This item was submitted to Loughborough's Research Repository by the author.

Items in Figshare are protected by copyright, with all rights reserved, unless otherwise indicated.

\title{
Robust autopilot design of uncertain bank-to-turn missiles using state-space disturbance observers
}

PLEASE CITE THE PUBLISHED VERSION

http://pig.sagepub.com/content/226/1/97

\section{PUBLISHER}

Sage Publications (ㅇ Institution of Mechanical Engineers (IMechE).

\section{VERSION}

AM (Accepted Manuscript)

\section{LICENCE}

CC BY-NC-ND 4.0

\section{REPOSITORY RECORD}

Yang, Jun, Wen-Hua Chen, and Shihua Li. 2012. "Robust Autopilot Design of Uncertain Bank-to-turn Missiles Using State-space Disturbance Observers”. figshare. https://hdl.handle.net/2134/11192. 
This item was submitted to Loughborough's Institutional Repository (https://dspace.lboro.ac.uk/) by the author and is made available under the following Creative Commons Licence conditions.

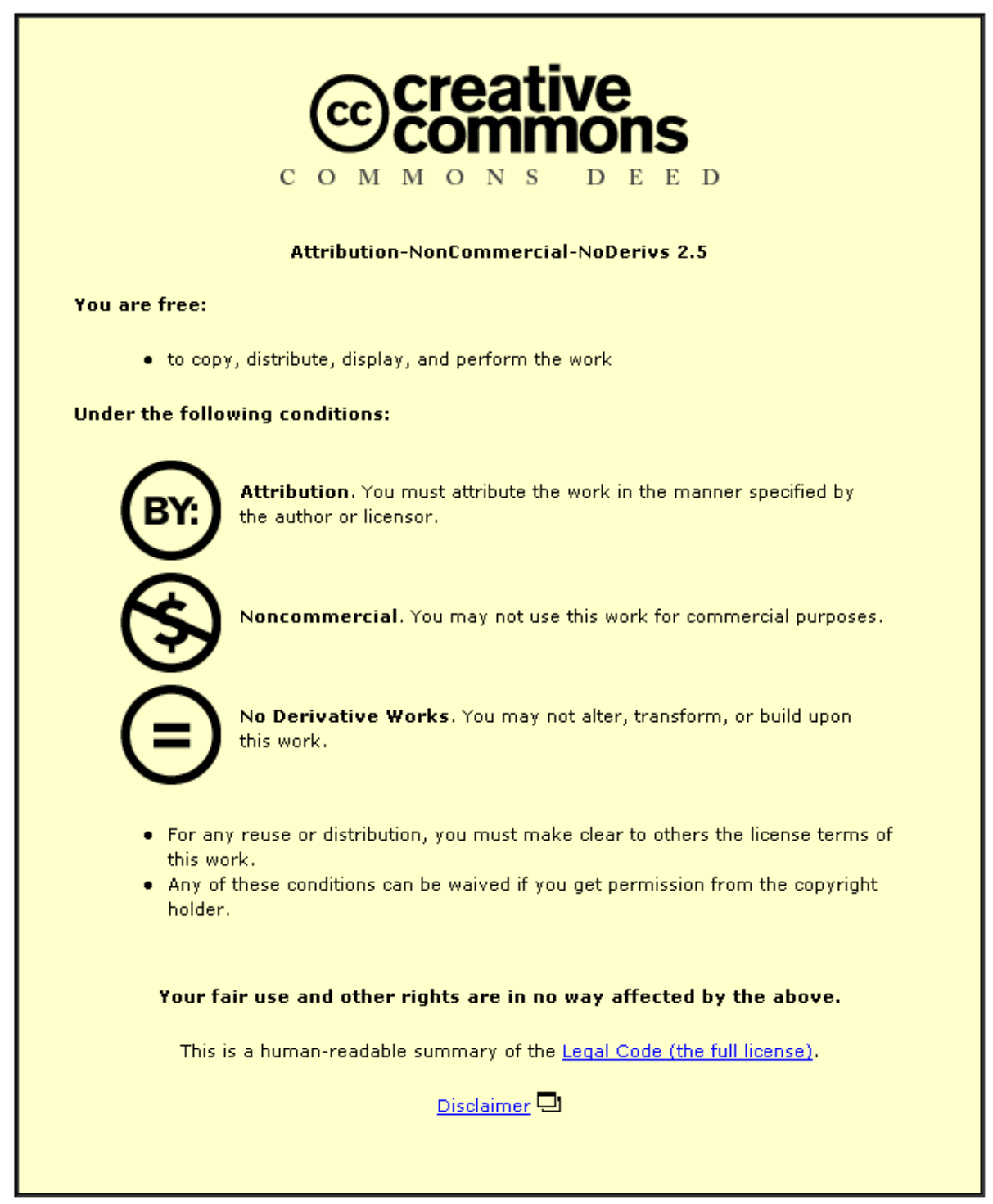

For the full text of this licence, please go to: http://creativecommons.org/licenses/by-nc-nd/2.5/ 


\title{
Robust autopilot design of uncertain bank-to-turn missiles using state-space disturbance observers
}

\author{
Jun Yang*, Wen-Hua Chen ${ }^{\dagger}$, and Shihua $\mathrm{Li}^{*}$
}

\begin{abstract}
Robust autopilot design for bank-to-turn (BTT) missiles under disturbances and uncertainties is investigated in this paper using the disturbance observer concept. It is well known that the BTT missile dynamics undergo a substantial changes during its flight. In this disturbance observer based control setting, the influences caused by parameter variations are merged into disturbance terms and regarded as parts of the lumped disturbances. Disturbance observers are employed to estimate the lumped disturbances and then a disturbance observer based robust control method is proposed in this paper to compensate the the influences of parameter variations and the disturbances from the output channels. Similar to the baseline LQR design, the disturbance observer based robust control is analysed and designed using linear techniques. Very promising performance has been achieved for the BTT missile as shown in simulation. It is demonstrated that disturbance observer based control approach provides a simple, intuitive and practical solution for many challenging control problems where systems are subject to significant external disturbances, and uncertainties such as BTT missiles.
\end{abstract}

Keywords: bank-to-turn missiles; pitch/yaw autopilot; disturbance observer; "mismatched" disturbances/uncertainties; external disturbances; parameter variations.

\section{Introduction}

Due to the growing interest in missiles with long range, high maneuverability and precision, the bank-to-turn (BTT) steering technique has become more and more popular as compared with the traditional skid-to-turn (STT) method $[1,2]$. By orienting the maximum aerodynamic normal force to the desired direction rapidly with a substantially large roll rate, the BTT missile exhibits many advantages over the STT missile, including high-lift, low-drag,

\footnotetext{
* J. Yang and S.H. Li are with School of Automation, Southeast University, Nanjing 210096, P.R. China, emails: junyang8402@gmail.com and 1sh@seu.edu.cn

${ }^{\dagger}$ W.-H. Chen is with Department of Aeronautical and Automotive Engineering, Loughborough University, LE11 3TU, UK, email: w.chen@lboro.ac.uk
} 
air-intake, internal carriage and increased range [1, 3, 4, 5]. However, the structure configuration of the BTT missile has undergone significant changes to take its advantages. These changes bring many challenges to autopilot designers, e.g., 1) the effects caused by timevarying parameters are more severe as compared with the STT style, 2) the high roll rate and the asymmetric structure will inevitably induce heavy nonlinear crossing couplings between different channels, and 3) the input/output dynamic characteristics for certain channels are nonminimum phase $[1,6]$.

To this end, autopilot design for BTT missiles has attracted extensive attentions, and various strategies have been proposed. Based on the models obtained by approximate linearization in given flight conditions or input/output feedback linearization technique, a few elegant control methods including robust control [3], gain-scheduling control [7, 8], nonlinear control [1], model predictive control [9], and switching control [10] have been employed for advanced autopilot designs. These methods are conceptually simple and can improve the performance from different aspects. However, difficult trade off has to be made between tracking performance, disturbance rejection and robustness against arodynamic changes in most of the above mentioned methods while others may not be able to take into aceeunt the

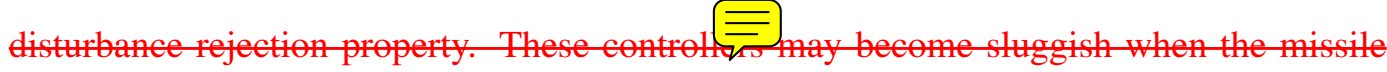
dynamies are highly nonlinear and undergo-significant parameter perturbations as well as strong external disturbances. To this end, developing active disturbance rejection approach is of great importance for the missile attopilot design. It is well known that feedforward control is an active approach in rejecting disturbances if the disturbance is measurable [11]. However, the uncertainties and disturbances in the missile systems are generally complicated and unmeasurable, thus an effective solution is to develop disturbance estimation techniques.

\section{Iplan to replace the above red part by the follow paragraph.}

However, difficult trade off is possibly required to be made between tracking perfor mance, disturbance rejection and robustness against aerodynamic changes in most of the above mentioned methods. These controllers may exhibit unsatisfactory performances when the missile dynamies are highly nonlinear and undergo significant parameter pertur bations as well as strong external disturbances. To this end, developing active disturbance rejection approach is of great impertance to improve the disturbance attenuation perfor mance of the missile autopilot.

By estimating and compensating the effects caused by disturbances/uncertainties, dis- 
turbance observer based control (DOBC) provides an active approach to handle system disturbances and improve robustness $[12,13,14,15]$ in the presence of unmeasurable uncertainties. In the past few decades, DOBC approaches have been successfully researched and applied in various engineering fields including robotic systems $[15,16]$, position systems $[17,18]$, grinding systems $[11,19]$ and flight control systems $[13,14,20,21]$. Compared with other robust control schemes, DOBC approach has two distinct features. One feature is that disturbance observer based compensation can be considered as a "patch" for existing controllers which may have unsatisfactory disturbance attenuation and robustness against uncertainties. The benefits of this are that there is no change to a baseline controller which may have been widely used and developed for many years. The second feature is that DOBC is not a worst-case based design. Most of the existing robust control methods are worst-case based design, and have been criticized as being "over conservative". In DOBC approach, the nominal performance of the baseline controller is recovered in the absence of disturbances or uncertainties.

In this paper, a disturbance observer based robust control (DOBRC) method is proposed to solve the disturbance attenuation problem of the BTT missile system. In the BTT missiles, the lumped disturbance torques caused by unmodeled dynamics, external winds, and parameter variations may affect the states directly rather than through the input channels, therefore don't satisfy "matching" conditions [22]. Using the DOBRC method, autopilot design of the BTT missile is carried out in this paper. The missile system under consideration is subject to "mismatched" disturbances including not only external disturbances, but also model uncertainties. The rest of the paper are organized as follows. The dynamic models of pitch/yaw channels of BTT missile are described in Section 2. In Section 3, disturbance observer design for BTT missile is investigated. The disturbance observer based robust control method is presented for the autopilot design in Section 4. Using the proposed method, simulation studies of the missile system are carried out in Section 5. The conclusions are finally given in Section 6.

\section{Pitch/yaw dynamic models of BTT missiles}

Since the roll rate in the BTT missile is much larger than the pitch and yaw rates, the pitch and yaw dynamics are severely interrupted by the roll dynamic. While the pitch and yaw dynamics have relatively smaller effects on the roll channel. To this end, a widely used method in the BTT missile is to design an autopilot for the pitch and yaw channels together, 
and design an autopilot for the roll channel separately [10,23]. In this paper, only autopilot design for pitch/yaw channels are considered as the roll channel design is relatively easy. The pitch/yaw dynamic models of the BTT missile are taken from [10, 23], depicted by

$$
\left\{\begin{aligned}
\dot{\omega}_{z}= & -\left(a_{1}+e_{1}\right) \omega_{z}+\left(e_{1} a_{4}-a_{2}\right) \alpha+\frac{e_{1}}{57.3} \omega_{x} \beta \\
& +\left(-e_{1} a_{5}-a_{3}\right) \delta_{z}+\frac{J_{x}-J_{y}}{57.3 J_{z}} \omega_{x} \omega_{y}+d_{\omega_{z}} \\
\dot{\alpha}= & \omega_{z}-\frac{1}{57.3} \omega_{x} \beta-a_{4} \alpha-a_{5} \delta_{z}+d_{\alpha} \\
\dot{\omega}_{y}= & -\left(b_{1}+e_{2}\right) \omega_{y}+\left(e_{2} b_{4}-b_{2}\right) \beta-\frac{e_{2}}{57.3} \omega_{x} \alpha \\
& +\left(e_{2} b_{5}-b_{3}\right) \delta_{y}+\frac{J_{z}-J_{x}}{57.3 J_{y}} \omega_{x} \omega_{z}+d_{\omega_{y}} \\
\dot{\beta}= & \omega_{y}+\frac{1}{57.3} \omega_{x} \alpha-b_{4} \beta-b_{5} \delta_{y}+d_{\beta} \\
n_{z}= & -\frac{b_{4} v_{t}}{57.3 g} \beta-\frac{b_{5} v_{t}}{57.3 g} \delta_{y}+d_{n_{z}} \\
n_{y}= & \frac{a_{4} v_{t}}{57.3 g} \alpha+\frac{a_{5} v_{t}}{57.3 g} \delta_{z}+d_{n_{y}}
\end{aligned}\right.
$$

where $\omega_{x}, \omega_{y}$ and $\omega_{z}$ are roll, yaw, and pitch rates, respectively. Variables $\alpha$ and $\beta$ represent the angle-of-attack and the sideslip angle, while $\delta_{y}$ and $\delta_{z}$ are yaw and pitch control deflection angles, respectively. $n_{z}$ and $n_{y}$ denote the overloads on the normal and side direction. $d_{\omega_{z}}, d_{\alpha}, d_{\omega_{y}}, d_{\beta}, d_{n_{z}}, d_{n_{y}}$ denote external disturbances on each equation. Parameters $J_{x}$, $J_{y}$, and $J_{z}$ denote roll, yaw and pitch moments of inertia, respectively. $v_{t}$ and $g$ are the instantaneous speed and the gravity acceleration. Coefficients $a_{i}, b_{i}(i=1,2, \cdots, 5), e_{1}$ and $e_{2}$ are aerodynamic parameters of the missile systems.

During the flight process of the missile, the aerodynamic parameters vary with the change of the missile height and velocity. In addition, the parameter perturbations are very complex, almost impossible to obtain their analytic forms. The aerodynamic parameters for different operating points are taken from [10] and listed in Table 1.

Since the roll rate is much larger than the pitch and yaw rates, it is reasonable to take $\omega_{x}$ as a parameter of the pitch/yaw models [23] and the following linear model is obtained by reformulating Eq.(1) as

$$
\left\{\begin{array}{c}
\dot{\boldsymbol{x}}=\mathbf{A} \boldsymbol{x}+\mathbf{B}_{u} \boldsymbol{u}+\mathbf{B}_{d} \boldsymbol{d}_{x} \\
\boldsymbol{y}=\mathbf{C} \boldsymbol{x}+\mathbf{D}_{u} \boldsymbol{u}+\mathbf{D}_{d} \boldsymbol{d}_{y}
\end{array}\right.
$$

with matrices

$$
\mathbf{A}=\left[\begin{array}{cccc}
-a_{1}-e_{1} & e_{1} a_{4}-a_{2} & \frac{\left(J_{x}-J_{y}\right) \omega_{x}}{57.3 J_{z}} & \frac{e_{1} \omega_{x}}{57.3} \\
1 & -a_{4} & 0 & \frac{-\omega_{x}}{57.3} \\
\frac{\left(J_{z}-J_{x}\right) \omega_{x}}{57.3 J_{y}} & \frac{-e_{2} \omega_{x}}{57.3} & -b_{1}-e_{2} & e_{2} b_{4}-b_{2} \\
0 & \frac{\omega_{x}}{57.3} & 1 & -b_{4}
\end{array}\right]
$$


Table 1: Aerodynamic parameters of a BTT missile for different operating points

\begin{tabular}{lllll} 
Operating points & $t_{1}(4.4 \mathrm{~s})$ & $t_{2}(11.7 \mathrm{~s})$ & $t_{3}(19.5 \mathrm{~s})$ & $t_{4}(23 \mathrm{~s})$ \\
\hline$a_{1}$ & 1.593 & 1.485 & 1.269 & 1.130 \\
$a_{2}$ & 260.559 & 266.415 & 196.737 & 137.385 \\
$a_{3}$ & 185.488 & 182.532 & 176.932 & 160.894 \\
$a_{4}$ & 1.506 & 1.295 & 1.169 & 1.130 \\
$a_{5}$ & 0.298 & 0.243 & 0.217 & 0.191 \\
$b_{1}$ & 1.655 & 1.502 & 1.269 & 1.130 \\
$b_{2}$ & 39.988 & -24.627 & -31.452 & -41.425 \\
$b_{3}$ & 159.974 & 170.532 & 182.030 & 184.093 \\
$b_{4}$ & 0.771 & 0.652 & 0.680 & 0.691 \\
$b_{5}$ & 0.254 & 0.191 & 0.188 & 0.182 \\
$e_{1}$ & 0.285 & 0.192 & 0.147 & 0.118 \\
$e_{2}$ & 0.295 & 0.195 & 0.147 & 0.118 \\
\hline
\end{tabular}

$$
\begin{aligned}
& \mathbf{B}_{u}=\left[\begin{array}{cc}
-e_{1} a_{5}-a_{3} & 0 \\
-a_{5} & 0 \\
0 & e_{2} b_{5}-b_{3} \\
0 & -b_{5}
\end{array}\right] \\
& \mathbf{C}=\left[\begin{array}{cccc}
0 & 0 & 0 & \frac{-b_{4} v_{t}}{57.3 g} \\
0 & \frac{a_{4} v_{t}}{57.3 g} & 0 & 0
\end{array}\right] \\
& \mathbf{D}_{u}=\left[\begin{array}{cc}
0 & \frac{-b_{5} v_{t}}{57.3 g} \\
\frac{a_{5} v_{t}}{57.3 g} & 0
\end{array}\right]
\end{aligned}
$$

$\mathbf{B}_{d}=\mathbf{I}_{4 \times 4}, \mathbf{D}_{d}=\mathbf{I}_{2 \times 2}$, the state vector $\boldsymbol{x}=\left[\omega_{z} \alpha \omega_{y} \beta\right]^{T}$, the control input $\boldsymbol{u}=\left[\delta_{z} \delta_{y}\right]^{T}$, the output $\boldsymbol{y}=\left[\begin{array}{ll}n_{z} & n_{y}\end{array}\right]^{T}$, the external disturbances on states $\boldsymbol{d}_{x}=\left[d_{\omega_{z}} d_{\alpha} d_{\omega_{y}} d_{\beta}\right]^{T}$ and outputs $\boldsymbol{d}_{y}=\left[d_{n_{z}} d_{n_{y}}\right]^{T}$.

The autopilot design objective is to achieve a good overload tracking performance according to the guidance commands as well as robustness against external disturbances and model uncertainties caused by parameter variations. In addition, from the viewpoint of engineering practice, the sideslip angle should be restricted as $|\beta|<5$ deg and the actuator deflection angles should be limited within a region $\left|\delta_{\max }\right| \leq 21 \mathrm{deg}[24]$. 


\section{Disturbance observers}

In the framework of the disturbance observer technique, not only external disturbances but also the influence caused by model uncertainties can be estimated and attenuated. To facilitate this, the model (2) is rewritten as

$$
\left\{\begin{array}{c}
\dot{\boldsymbol{x}}=\mathbf{A}_{n} \boldsymbol{x}+\mathbf{B}_{n} \boldsymbol{u}+\mathbf{B}_{l d} \boldsymbol{d}_{l x} \\
\boldsymbol{y}=\mathbf{C}_{n} \boldsymbol{x}+\mathbf{D}_{n} \boldsymbol{u}+\mathbf{D}_{l d} \boldsymbol{d}_{l y}
\end{array}\right.
$$

where $\mathbf{A}_{n}, \mathbf{B}_{n}, \mathbf{C}_{n}$ and $\mathbf{D}_{n}$ are system matrices in the nominal case, $\boldsymbol{d}_{l x}$ and $\boldsymbol{d}_{l y}$ denote the lumped disturbances on states and outputs. Letting $\mathbf{B}_{l d}=\mathbf{I}_{4 \times 4}$ and $\mathbf{D}_{l d}=\mathbf{I}_{2 \times 2}$ and comparing (2) with (7) yields

$$
\begin{array}{r}
\boldsymbol{d}_{l x}=\mathbf{B}_{d} \boldsymbol{d}_{x}+\left(\mathbf{A}-\mathbf{A}_{n}\right) \boldsymbol{x}+\left(\mathbf{B}-\mathbf{B}_{n}\right) \boldsymbol{u} \\
\boldsymbol{d}_{l y}=\mathbf{D}_{d} \boldsymbol{d}_{y}+\left(\mathbf{C}-\mathbf{C}_{n}\right) \boldsymbol{x}+\left(\mathbf{D}-\mathbf{D}_{n}\right) \boldsymbol{u}
\end{array}
$$

The lumped disturbances consist of external disturbances and internal disturbances caused by model uncertainties. Disturbance observers are now designed to estimate the disturbances $\boldsymbol{d}_{l x}$ and $\boldsymbol{d}_{l y}$ using the input, output and state information. The estimate of $\boldsymbol{d}_{l y}$ can be obtained from the second equation in (7), described by

$$
\hat{\boldsymbol{d}}_{l y}=\boldsymbol{y}-\mathbf{C}_{n} \boldsymbol{x}-\mathbf{D}_{n} \boldsymbol{u}
$$

Combining (7) with (10), the estimation error $\boldsymbol{e}_{d_{l y}}$ can be obtained as

$$
\boldsymbol{e}_{d_{l y}}=\hat{\boldsymbol{d}}_{l y}-\boldsymbol{d}_{l y}=\mathbf{0}
$$

Assumption 1: Suppose that the lumped disturbances $\boldsymbol{d}_{l x}$ varies slowly relative to the observer dynamics and has constant steady-state values $\lim _{t \rightarrow \infty} \dot{\boldsymbol{d}}_{l x}(t)=0$ or $\lim _{t \rightarrow \infty} \boldsymbol{d}_{l x}(t)=$ $\boldsymbol{d}_{l x s}$.

Remark 1: The results in this paper are based on Assumption 1. However, it is shown that the method is also feasible for fast time-varying disturbances [15].

Remark 2: In the presence of uncertainties, the lumped disturbances would be a function of the states, which can be reasonably estimated if the disturbance observer dynamics are faster than that of the closed-loop system. The same argument for the state observer based control methods is valid.

For system (7), the following observer is designed to estimate the disturbances $\boldsymbol{d}_{l x}$

$$
\left\{\begin{array}{l}
\dot{z}=-\mathbf{L}(z+\mathbf{L} \boldsymbol{x})-\mathbf{L}\left(\mathbf{A}_{n} \boldsymbol{x}+\mathbf{B}_{n} \boldsymbol{u}\right) \\
\hat{\boldsymbol{d}}_{l x}=\boldsymbol{z}+\mathbf{L} \boldsymbol{x}
\end{array}\right.
$$


where $\hat{\boldsymbol{d}}_{l x}$ is the estimate of the lumped disturbance on the states $\boldsymbol{d}_{l x}, \boldsymbol{z}$ is an auxiliary vector and $\mathbf{L}$ is the observer gain matrix to be designed.

Lemma 1 [25]: Consider system (7) under the lumped disturbances which satisfy Assumption 1. The estimates of the disturbance observer (12) asymptotically tracks the lumped disturbances if the observer gain matrix $\mathbf{L}$ is chosen such that $-\mathbf{L}$ is a Hurwitz matrix.

Proof: The disturbance estimation error of the DOB (12) is defined as

$$
\boldsymbol{e}_{d_{l x}}=\hat{\boldsymbol{d}}_{l x}-\boldsymbol{d}_{l x}
$$

Combining (7), (12) with (13) gives

$$
\begin{aligned}
\dot{\boldsymbol{e}}_{d_{l x}}= & \dot{\hat{\boldsymbol{d}}}_{l x}-\dot{\boldsymbol{d}}_{l x} \\
= & \dot{\boldsymbol{z}}+\mathbf{L} \dot{\boldsymbol{x}}-\dot{\boldsymbol{d}}_{l x} \\
= & -\mathbf{L} \hat{\boldsymbol{d}}_{l x}-\mathbf{L}\left(\mathbf{A}_{n} \boldsymbol{x}+\mathbf{B}_{n} \boldsymbol{u}\right) \\
& +\mathbf{L}\left(\mathbf{A}_{n} \boldsymbol{x}+\mathbf{B}_{n} \boldsymbol{u}+\boldsymbol{d}_{l x}\right)-\dot{\boldsymbol{d}}_{l x} \\
= & -\mathbf{L}\left(\hat{\boldsymbol{d}}_{l x}-\boldsymbol{d}_{l x}\right)-\dot{\boldsymbol{d}}_{l x}=-\mathbf{L} \boldsymbol{e}_{d_{l x}}-\dot{\boldsymbol{d}}_{l x}
\end{aligned}
$$

Since all eigenvalues of matrix $-\mathbf{L}$ are in the left hand side of the complex plane, Eq. (14) is asymptotically stable. This implies that the estimate of DOB can track the disturbances asymptotically under the condition that $\lim _{t \rightarrow \infty} \dot{\boldsymbol{d}}_{l x}(t)=\boldsymbol{0}_{4 \times 1}$.

Since $\mathbf{B}_{n} \neq \mathbf{I}_{4 \times 4}, \mathbf{D}_{n} \neq \mathbf{I}_{2 \times 2}$, it can be found from Eq.(7) that the disturbances and uncertainties are "mismatched" ones. The existing DOBC methods are not applicable in such a case.

It should be pointed out that the "mismatched" disturbances can not be attenuated from the state equations generally. In this paper, based on the disturbance estimate of DOB, the composite control law like $\boldsymbol{u}=\mathbf{K}_{x} \boldsymbol{x}+\mathbf{K}_{d} \hat{\boldsymbol{d}}$ is designed and we attempt to find an appropriate $\mathbf{K}_{d}$ to assure that the disturbances can be removed from the output channel in steady-state.

\section{Disturbance observer based robust control}

\subsection{Feedback control design}

In the proposed composite DOBRC method, any feedback controller which can stabilize system (7) and provide adequate overload tracking performance in the absence of disturbances and uncertainties can be adopted. The classical linear quadratic regulator (LQR) is considered as the baseline autopilot design for the BTT missiles. The penalty matrices $\mathbf{Q}$ 
and $\mathbf{R}$ in the cost function of LQR are selected as

$$
\mathbf{Q}=\left[\begin{array}{llll}
1 & 0 & 0 & 0 \\
0 & 1 & 0 & 0 \\
0 & 0 & 1 & 0 \\
0 & 0 & 0 & 1
\end{array}\right], \mathbf{R}=\left[\begin{array}{cc}
70 & 0 \\
0 & 70
\end{array}\right]
$$

The corresponding LQR feedback gain is obtained as

$$
\mathbf{K}_{x}=\left[\begin{array}{cccc}
0.097 & -0.278 & 0.004 & -0.066 \\
0.004 & -0.086 & 0.125 & 0.405
\end{array}\right]
$$

As shown in Figs. 2-4, a satisfactory step response is obtained (at the beginning of the responses).

\subsection{Stability analysis of the closed-loop system}

Different from all previous DOBC methods, our new DOBRC law for system (7) is designed as

$$
\boldsymbol{u}=\mathbf{K}_{x} \boldsymbol{x}+\mathbf{K}_{d} \hat{\boldsymbol{d}}
$$

where $\mathbf{K}_{d}=\left[\mathbf{K}_{d x} \mathbf{K}_{d y}\right]$, and $\hat{\boldsymbol{d}}=\left[\hat{\boldsymbol{d}}_{l x} \hat{\boldsymbol{d}}_{l y}\right]^{T}$.

Assumption 2: The lumped disturbances satisfy that $\boldsymbol{d}_{l x}, \boldsymbol{d}_{l y}$ and $\dot{\boldsymbol{d}}_{l x}$ are bounded.

Lemma 2 [25]: Suppose that Assumption 2 is satisfied. The closed-loop system consisting of the plant (7), the DOB (12) and the composite control law (17) is bounded-inputbounded-output (BIBO) stable if the feedback control gain $\mathbf{K}_{x}$ and the observer gain matrix $\mathbf{L}$ are selected such that 1 ) the system (7) in the absence of disturbances under control law $\boldsymbol{u}=\mathbf{K}_{x} \boldsymbol{x}$ is asymptotically stable, and 2) the disturbance estimate by DOB can track the disturbance asymptotically.

Proof: Combining system (7) with the DOBRC law (17) and disturbance observers (10)-(13), the closed-loop system is obtained as

$$
\begin{aligned}
{\left[\begin{array}{c}
\dot{\boldsymbol{x}} \\
\dot{\boldsymbol{e}}_{d_{l x}} \\
\dot{\boldsymbol{e}}_{d_{l y}}
\end{array}\right]=} & {\left[\begin{array}{ccc}
\mathbf{A}_{n}+\mathbf{B}_{n} \mathbf{K}_{x} & \mathbf{B}_{n} \mathbf{K}_{d x} & \mathbf{B}_{n} \mathbf{K}_{d y} \\
\mathbf{O} & -\mathbf{L} & \mathbf{O} \\
\mathbf{O} & \mathbf{O} & \mathbf{O}
\end{array}\right]\left[\begin{array}{c}
\boldsymbol{x} \\
\boldsymbol{e}_{d_{l x}} \\
\boldsymbol{e}_{d_{l y}}
\end{array}\right] } \\
+ & {\left[\begin{array}{ccc}
\mathbf{I}_{4 \times 4}+\mathbf{B}_{n} \mathbf{K}_{d x} & \mathbf{B}_{n} \mathbf{K}_{d y} & \mathbf{O} \\
\mathbf{O} & \mathbf{O} & -\mathbf{I}_{4 \times 4} \\
\mathbf{O} & \mathbf{O} & \mathbf{O}
\end{array}\right]\left[\begin{array}{c}
\boldsymbol{d}_{l x} \\
\boldsymbol{d}_{l y} \\
\dot{\boldsymbol{d}}_{l x}
\end{array}\right] }
\end{aligned}
$$


Considering the condition $\boldsymbol{e}_{d_{l y}}=\boldsymbol{0}$ for all $t \geq 0$ in (11), the closed-loop system in (18) reduces to

$$
\begin{aligned}
{\left[\begin{array}{c}
\dot{\boldsymbol{x}} \\
\dot{\boldsymbol{e}}_{d_{l x}}
\end{array}\right] } & =\left[\begin{array}{cc}
\mathbf{A}_{n}+\mathbf{B}_{n} \mathbf{K}_{x} & \mathbf{B}_{n} \mathbf{K}_{d x} \\
\mathbf{O} & -\mathbf{L}
\end{array}\right]\left[\begin{array}{c}
\boldsymbol{x} \\
\boldsymbol{e}_{d_{l x}}
\end{array}\right] \\
& +\left[\begin{array}{cc}
\mathbf{I}_{4 \times 4}+\mathbf{B}_{n} \mathbf{K}_{d x} & \mathbf{O} \\
\mathbf{O} & -\mathbf{I}_{4 \times 4}
\end{array}\right]\left[\begin{array}{c}
\boldsymbol{d}_{l x} \\
\dot{\boldsymbol{d}}_{l x}
\end{array}\right]
\end{aligned}
$$

Conditions 1) and 2) imply that both $\mathbf{A}_{n}+\mathbf{B}_{n} \mathbf{K}_{x}$ and $-\mathbf{L}$ are Hurwitz matrices. To this end, it can be proved that

$$
\left[\begin{array}{cc}
\mathbf{A}_{n}+\mathbf{B}_{n} \mathbf{K}_{x} & \mathbf{B}_{n} \mathbf{K}_{d x} \\
\mathbf{O} & -\mathbf{L}
\end{array}\right]
$$

is also a Hurwitz matrix.

Moreover, Assumption 2 implies that the inputs of the closed-loop system (19) $\boldsymbol{d}_{l x}$ and $\dot{\boldsymbol{d}}_{l x}$ are bounded. This completes the proof that the outputs of the closed-loop system are bounded.

Lemma 3 [25]: Suppose that Both Assumption 1 and 2 are satisfied. The state vector $\boldsymbol{x}$ converges to a constant vector $\boldsymbol{x}_{s}=-\left(\mathbf{A}_{n}+\mathbf{B}_{n} \mathbf{K}_{x}\right)^{-1}\left(\mathbf{I}_{4 \times 4}+\mathbf{B}_{n} \mathbf{K}_{x}\right) \boldsymbol{d}_{l x s}$ asymptotically if the feedback control gain $\mathbf{K}_{x}$ and the observer gain matrix $\mathbf{L}$ are selected such that 1) the system (7) in the absence of disturbances under control law $\boldsymbol{u}=\mathbf{K}_{x} \boldsymbol{x}$ is asymptotically stable, and 2) the disturbance estimate by DOB can track the disturbance asymptotically.

Proof: Define the state error as

$$
\boldsymbol{e}_{x}=\boldsymbol{x}-\boldsymbol{x}_{s}
$$

Combining (19) and (20), the closed-loop system is given by

$$
\begin{gathered}
{\left[\begin{array}{c}
\dot{\boldsymbol{e}}_{x} \\
\dot{\boldsymbol{e}}_{d_{l x}}
\end{array}\right]=\left[\begin{array}{cc}
\mathbf{A}_{n}+\mathbf{B}_{n} \mathbf{K}_{x} & \mathbf{B}_{n} \mathbf{K}_{d x} \\
\mathbf{O} & -\mathbf{L}
\end{array}\right]\left[\begin{array}{c}
\boldsymbol{e}_{x} \\
\boldsymbol{e}_{d_{l x}}
\end{array}\right]} \\
+\left[\begin{array}{cc}
\mathbf{I}_{4 \times 4}+\mathbf{B}_{n} \mathbf{K}_{d x} & \mathbf{O} \\
\mathbf{O} & -I_{4 \times 4}
\end{array}\right]\left[\begin{array}{c}
\boldsymbol{d}_{l x}-\boldsymbol{d}_{l x s} \\
\dot{\boldsymbol{d}}_{l x}
\end{array}\right]
\end{gathered}
$$

Similar with the proof of Lemma 2, it can be proved that the system matrix of (21) is Hurwitz. Assumption 1 implies that $\lim _{t \rightarrow \infty} \dot{\boldsymbol{d}}_{l x}(t)=\boldsymbol{0}$ and $\lim _{t \rightarrow \infty}\left[\boldsymbol{d}_{l x}(t)-\boldsymbol{d}_{l x s}\right]=\boldsymbol{0}$. To this end, the closed-loop system governed by (21) is asymptotically stable. This implies that $\lim _{t \rightarrow \infty} \boldsymbol{e}_{x}(t)=\boldsymbol{0}$. This completes the proof. 


\subsection{Design of the disturbance compensation gain}

The main contribution of this work is investigating how to design the disturbance compensation gain $\mathbf{K}_{d}$ such that the effects caused by the "mismatched" disturbances can be attenuated from the output channels asymptotically.

Theorem 1: Suppose that disturbances in system (7) satisfy Assumption 1 and 2, and the feedback control gain $\mathbf{K}_{x}$ and the observer gain matrix $\mathbf{L}$ are selected such that $\mathbf{A}_{n}+$ $\mathbf{B}_{n} \mathbf{K}_{x}$ and $-\mathbf{L}$ are Hurwitz matrices. The disturbances applied on the system (7) can be asymptotically attenuated from the output channel by the newly designed DOBRC law (17) if the disturbance compensation gains in $\mathbf{K}_{d}$ are selected as

$$
\begin{gathered}
\mathbf{K}_{d x}=\left[\mathbf{D}_{n}-\left(\mathbf{C}_{n}+\mathbf{D}_{n} \mathbf{K}_{x}\right)\left(\mathbf{A}_{n}+\mathbf{B}_{n} \mathbf{K}_{x}\right)^{-1} \mathbf{B}_{n}\right]^{-1} \\
\times\left(\mathbf{C}_{n}+\mathbf{D}_{n} \mathbf{K}_{x}\right)\left(\mathbf{A}_{n}+\mathbf{B}_{n} \mathbf{K}_{x}\right)^{-1} \mathbf{B}_{l d} \\
\mathbf{K}_{d y}=-\left[\mathbf{D}_{n}-\left(\mathbf{C}_{n}+\mathbf{D}_{n} \mathbf{K}_{x}\right)\left(\mathbf{A}_{n}+\mathbf{B}_{n} \mathbf{K}_{x}\right)^{-1} \mathbf{B}_{n}\right]^{-1} \mathbf{D}_{l d}
\end{gathered}
$$

Proof: By substituting the control law (17) into system (7), the state is expressed as

$$
\boldsymbol{x}=\left(\mathbf{A}_{n}+\mathbf{B}_{n} \mathbf{K}_{x}\right)^{-1}\left[\dot{\boldsymbol{x}}-\mathbf{B}_{n} \mathbf{K}_{d} \hat{\boldsymbol{d}}-\mathbf{B}_{l d} \boldsymbol{d}_{l x}\right]
$$

Combining (7), (22), (23), with (24) gives

$$
\begin{aligned}
\boldsymbol{y}= & \left(\mathbf{C}_{n}+\mathbf{D}_{n} \mathbf{K}_{x}\right)\left(\mathbf{A}_{n}+\mathbf{B}_{n} \mathbf{K}_{x}\right)^{-1} \dot{\boldsymbol{x}} \\
& +\left(\mathbf{D}_{n}-\left(\mathbf{C}_{n}+\mathbf{D}_{n} \mathbf{K}_{x}\right)\left(\mathbf{A}_{n}+\mathbf{B}_{n} \mathbf{K}_{x}\right)^{-1} \mathbf{B}_{n}\right) \mathbf{K}_{d} \hat{\boldsymbol{d}} \\
& -\left(\mathbf{C}_{n}+\mathbf{D}_{n} \mathbf{K}_{x}\right)\left(\mathbf{A}_{n}+\mathbf{B}_{n} \mathbf{K}_{x}\right)^{-1} \mathbf{B}_{l d} \boldsymbol{d}_{l x}+\mathbf{D}_{l d} \boldsymbol{d}_{l y} \\
= & \left(\mathbf{C}_{n}+\mathbf{D}_{n} \mathbf{K}_{x}\right)\left(\mathbf{A}_{n}+\mathbf{B}_{n} \mathbf{K}_{x}\right)^{-1} \dot{\boldsymbol{x}}+ \\
& \left(\mathbf{C}_{n}+\mathbf{D}_{n} \mathbf{K}_{x}\right)\left(\mathbf{A}_{n}+\mathbf{B}_{n} \mathbf{K}_{x}\right)^{-1} \mathbf{B}_{l d} \boldsymbol{e}_{l l x}-\mathbf{D}_{l d} \boldsymbol{e}_{l l y}
\end{aligned}
$$

According to Lemma 3, it can be concluded that

$$
\lim _{t \rightarrow \infty} \dot{\boldsymbol{x}}(t)=\boldsymbol{0}, \lim _{t \rightarrow \infty} \boldsymbol{e}_{d_{l x}}(t)=\mathbf{0}
$$

It can be deduced form (11), (25) and (26) that

$$
\lim _{t \rightarrow \infty} \boldsymbol{y}(t)=\boldsymbol{0}
$$

Remark 3: Note that the disturbance compensation gain $\mathbf{K}_{d}$ in (22) and (23) is a general case and suitable for both "matched" and "mismatched" disturbances. In "matched" case, i.e., $\mathbf{B}_{n}=\mathbf{B}_{l d}$ and $\mathbf{D}_{n}=\mathbf{D}_{l d}=0$, it can be obtained from (22) and (23) that the disturbance 
compensation gain is reduced to $\mathbf{K}_{d x}=-\mathbf{I}$ and $\mathbf{K}_{d y}=\mathbf{O}$ which is the particular form in the previous literature $[14,15]$.

In our work, the observer gain matrix in DOB (12) is selected as

$$
\mathbf{L}=\left[\begin{array}{cccc}
50 & 0 & 0 & 0 \\
0 & 50 & 0 & 0 \\
0 & 0 & 50 & 0 \\
0 & 0 & 0 & 50
\end{array}\right]
$$

The disturbance compensation gains can also be calculated by Eqs. (22) and (23), given as

$$
\begin{gathered}
\mathbf{K}_{d x}=\left[\begin{array}{cccc}
0.007 & 0.099 & 0.001 & 0.052 \\
-0.001 & -0.047 & 0.007 & 0.129
\end{array}\right] \\
\mathbf{K}_{d y}=\left[\begin{array}{cc}
-0.516 & 0.450 \\
-0.332 & -0.528
\end{array}\right]
\end{gathered}
$$

The control structure of the proposed DOBRC for the BTT missile system is shown in Fig. 1.

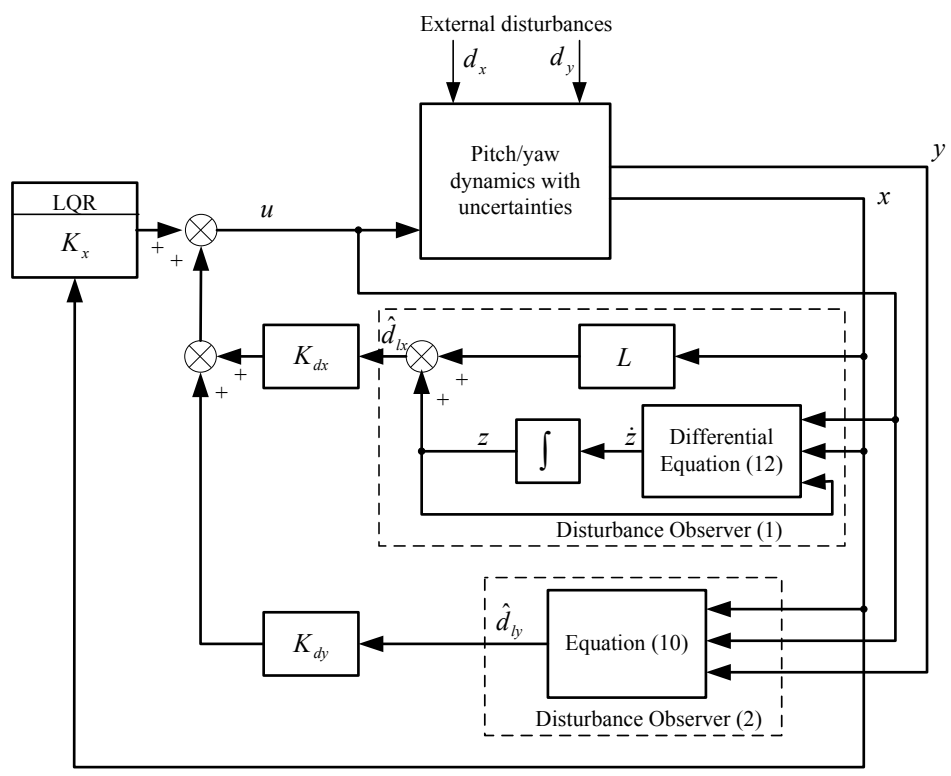

Figure 1: Block diagram of the proposed DOBRC method for the BTT missile system.

\section{Simulation studies}

In this section, both external disturbances and model uncertainties are considered to show the effectiveness of the proposed DOBRC methods. In the simulation, it is supposed that 
the missile travels with a velocity of $v_{t}=1000 \mathrm{~m} / \mathrm{s}$ and a roll rate of $\omega_{x}=400 \mathrm{deg} / \mathrm{s}$. To demonstrate the efficiency of the proposed method in improving external disturbance rejection performance and robustness against model uncertainties, the baseline LQR controller is employed for the purpose of comparison.

\subsection{External disturbance rejection performance}

Suppose that step external disturbances are imposed on each equation in (1), actually, $d_{\omega_{z}}=$ $d_{\alpha}=d_{\omega_{y}}=d_{\beta}=4$ and $d_{n_{z}}=d_{n_{y}}=1$ at $t=2 \mathrm{sec}$. The output responses and input profiles of the pitch/yaw channels under the proposed DOBRC method are shown in Figs. 2 and 3, respectively. The corresponding responses of the states are shown in Fig. 4.

(a)

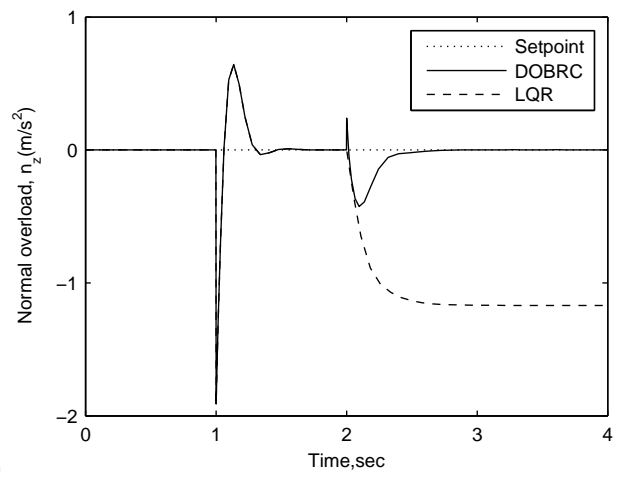

(b)

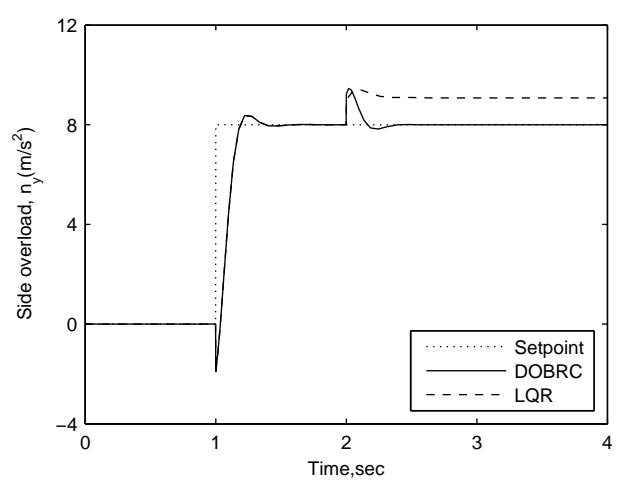

Figure 2: Output responses in the presence of external disturbances under the proposed DOBRC (solid line) and the LQR (dashed line): (a) normal overload, $n_{z}$; (b) side overload, $n_{y}$. The reference signals are denoted by dotted lines.

(a)

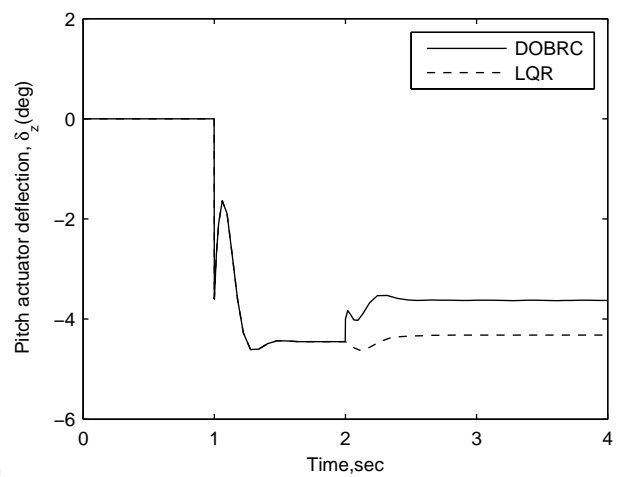

(b)

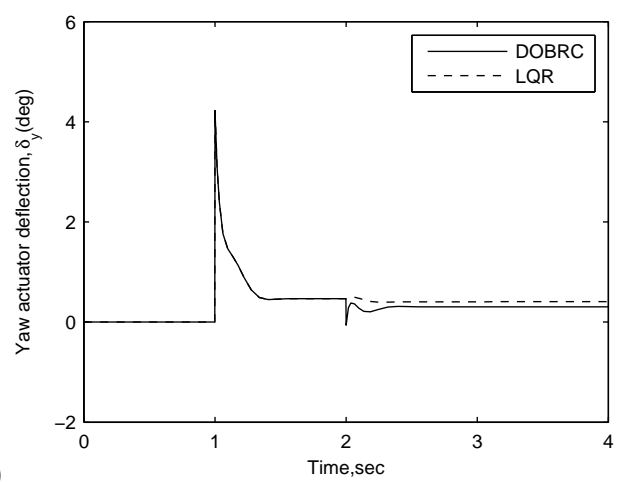

Figure 3: Input time histories in the presence of external disturbances under the proposed DOBRC (solid line) and the LQR (dashed line): (a) pitch actuator deflection, $\delta_{z}$; (b) yaw actuator deflection, $\delta_{y}$. 

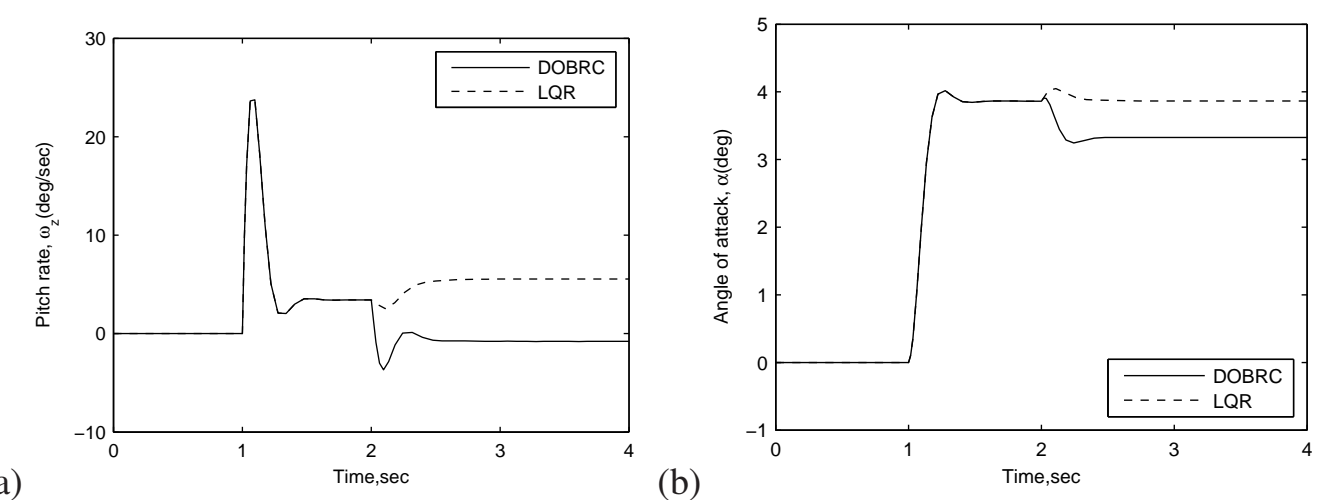

(a)

(b)
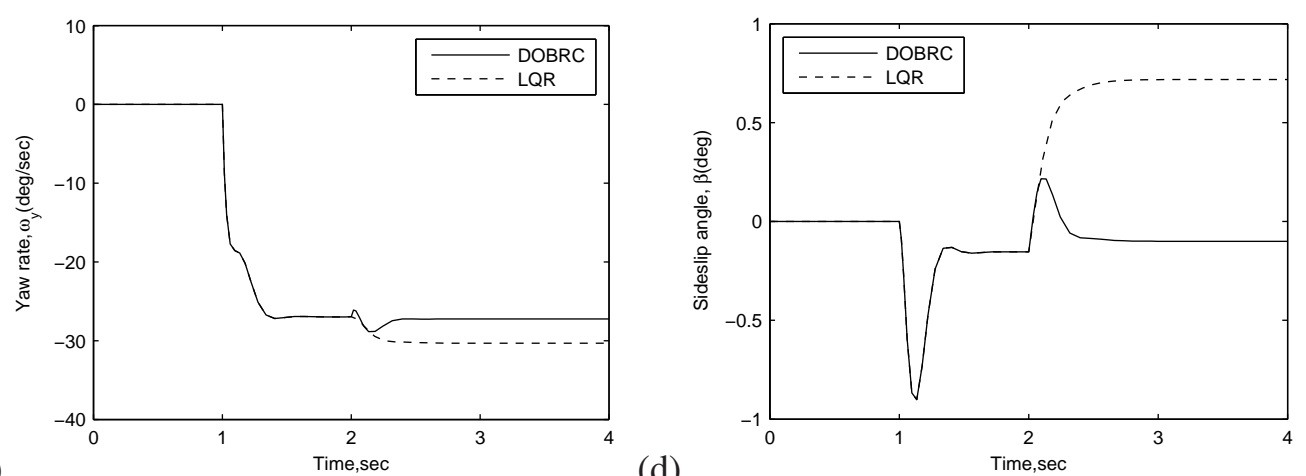

(d)

Figure 4: State responses in the presence of external disturbances under the proposed DOBRC (solid line) and the LQR (dashed line): (a) pitch rate, $\omega_{z}$; (b) angle of attack, $\alpha$; (c) yaw rate, $\omega_{y} ;$ (d) sideslip angle, $\beta$.

As shown in Figs. 2-4, in the first two seconds, the control performance under the proposed method recovered to that under the baseline LQR controller since there are no disturbances during this time period. It can be observed from Figs. 2-4 that, when the disturbances appear, the feedforward part of the proposed method which serving as a "patch" to the baseline controller becomes active in rejecting the disturbances, while the baseline LQR controller fails to effectively counteract the disturbances. This implies that the nominal performance of the proposed method is preserved in the absence of the external disturbances. In the presence of the "mismathced" external disturbances, an excellent disturbance rejection as indicated by a short settling time and offset-free in steady state exhibits by the proposed DOBRC approach. Therefore a good disturbance rejection ability is achieved without sacrificing the nominal performance, which is one of the major advantages of the proposed method. Furthermore, Fig. 3 shows that the actuator deflection angles are within the allowable regions and no excessive control energy is required. 


\subsection{Robustness against model uncertainties}

As listed in Table 1, the aerodynamic parameters are time-varying during the missile flies. To representing the continuous changes of the real missile dynamics during its flight, a linear transition of the parameters from one operating points to another happens [26] is adopted. For instance, aerodynamic parameters $a_{i}(t), i=1,2, \cdots, 5$, are expressed as

$$
a_{i}(t)=\left\{\begin{array}{l}
a_{i}\left(t_{1}\right),\left(0 \leq t<t_{1}\right), \\
a_{i}\left(t_{j-1}\right)+\frac{a_{i}\left(t_{j}\right)-a_{i}\left(t_{j-1}\right)}{t_{j}-t_{j-1}}\left(t-t_{j-1}\right), \\
\left(t_{j-1} \leq t<t_{j}(j=2,3, \cdots, 7)\right) .
\end{array}\right.
$$

The parameters $b_{i}(t), i=1,2, \cdots, 5$, and $e_{i}(t), i=1,2$, change in a similar form with the time as described by the formula (31).

In the presence of such model uncertainties caused by time-varying parameters, the response curves of the outputs, inputs and states under the baseline LQR design and the DOBRC approach are compared in Figs. 5, 6 and 7, respectively.

(a)

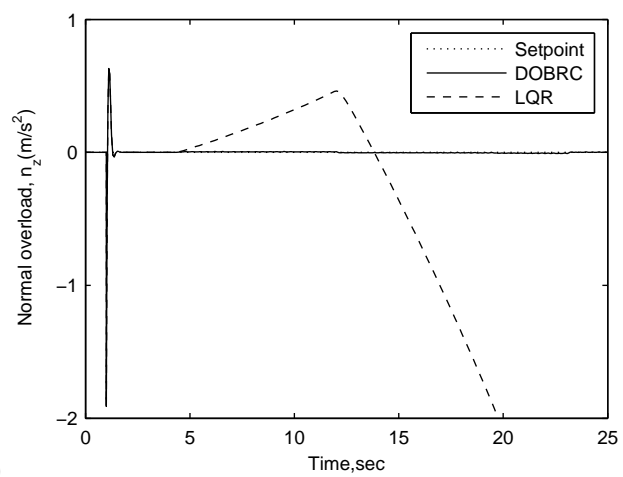

(b)

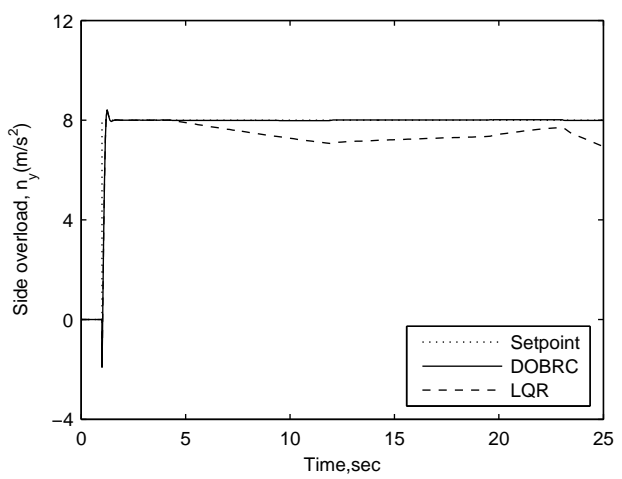

Figure 5: Output responses in the presence of model uncertainties under the proposed DOBRC (solid line) and the LQR (dashed line): (a) normal overload, $n_{z}$; (b) side overload, $n_{y}$. The reference signals are denoted by dotted lines.

At time $t=1$ second, a step command is applied on the side overload. Again both the baseline LQR controller and the proposed DOBRC cope with this quite well. Due to the coupling between the pitch and yaw channels, a quite significant normal acceleration is generated but the $\mathrm{LQR}$ and DOBRC regulate the normal acceleration to zero as required quite quickly. As the missile undergoes continuous change of its dynamics, gradually a large error has been built up in LQR design, which indicates that the LQR has a good robustness when the model error is within a certain range but poor performance even instability may be experienced in the presence of a quite substantial uncertainty. In contrast, the DOBRC 

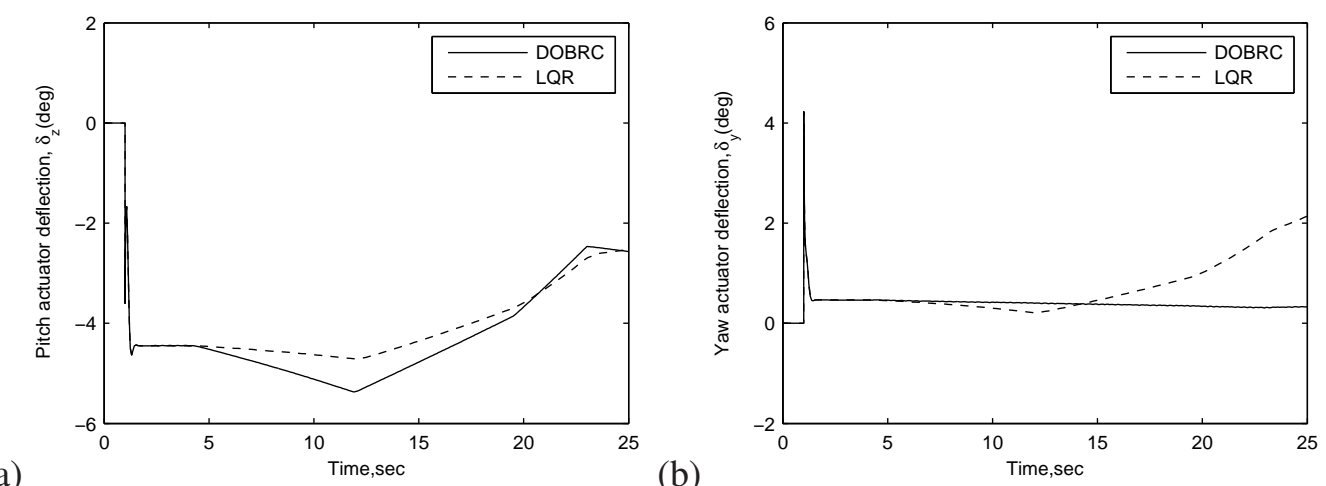

Figure 6: Input time histories in the presence of model uncertainties under the proposed DOBRC (solid line) and the LQR (dashed line): (a) pitch actuator deflection, $\delta_{z}$; (b) yaw actuator deflection, $\delta_{y}$.

approach exhibits an excellent robustness performance. It can be observed from Fig. 5 that the output responses can precisely track the reference commands during the whole missile flight regardless of the substantial changes of its parameters. To achieve this, as shown in Fig. 6, the pitch control $\delta_{z}$ continuously varies to compensate the influence of the parameter variations. Fig. 7 shows that the yaw rate $\omega_{y}$ experiences a large change during the flight, which may be caused by severe aerodynamic parameter perturbations. It shall be highlighted that the sideslip angle and the actuator deflections are well within the specified ranges. Therefore, it is concluded from this simulation exercise that the baseline LQR controller exhibits unsatisfactory control performance in this case, while the proposed method achieves surprisingly promising robustness against model uncertainties caused by substantial parameter variations. The proposed DORBC method provides a promising way to improve the robustness of the baseline controller without resorting to a high gain design as in many robust control methods.

\section{Conclusion}

Using a newly proposed disturbance observer based robust control method, the disturbance attenuation problem of a BTT missile has been investigated in this paper. The "disturbance" under consideration is a general concept which may include both external disturbances and internal disturbances caused by parameter variations. In this setting, the disturbance generally does not satisfy the "matching" condition, i.e., the disturbances enter the system with different channels from the control inputs. A new DOBRC method has been proposed 


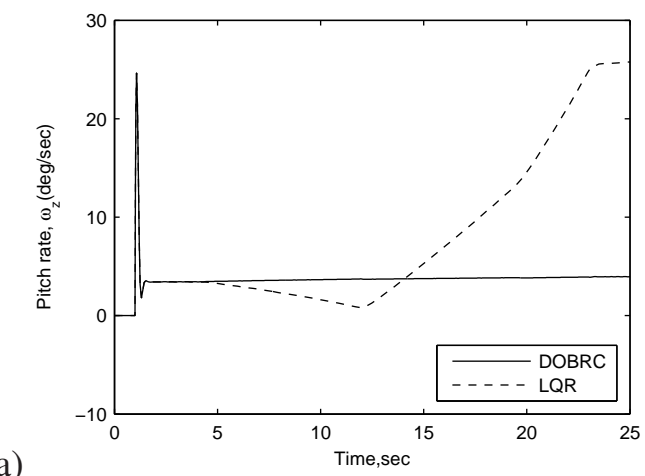

(a)

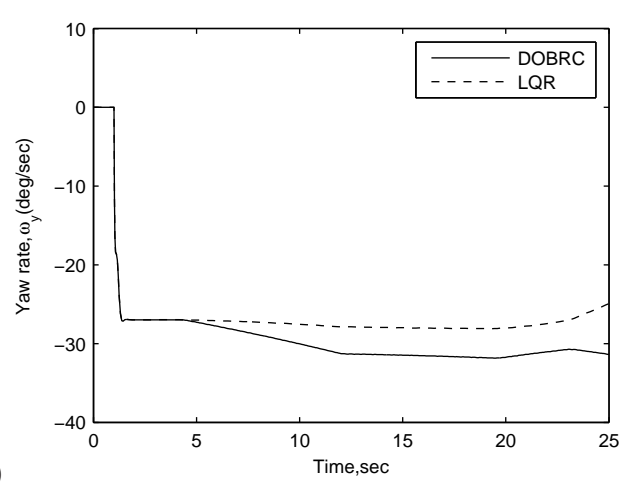

(b)
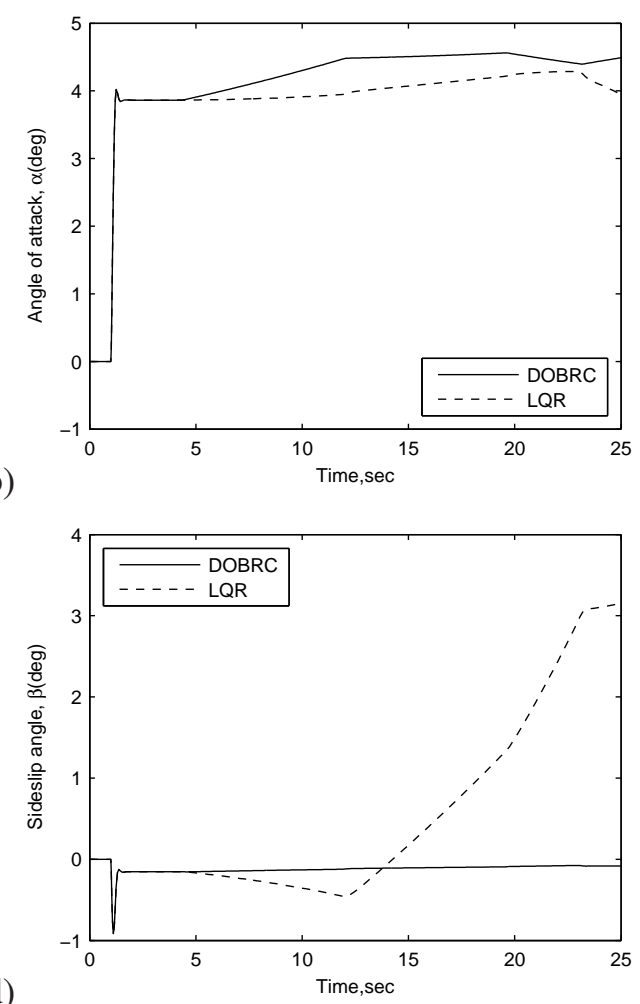

Figure 7: State responses in the presence of model uncertainties under the proposed DOBRC (solid line) and the LQR (dashed line): (a) pitch rate, $\omega_{z}$; (b) angle of attack, $\alpha$; (c) yaw rate, $\omega_{y}$; (d) sideslip angle, $\beta$.

for the BTT missile to improve its disturbance attenuation and in particular the robustness against the substantial variations of the parameters during the flight. It should be pointed out that the proposed method is very easy for practical implementation since only linear analysis and synthesis approaches are employed. The simulation results have demonstrated that very promising disturbance attenuation and strong robustness have been achieved by the proposed disturbance observer based approach. The results in this paper show that the disturbance observer based control technique provides a very promising, practical solution for challenging control problems with large uncertainties and significant external disturbanc. Its concept is quite intuitive and practical without resorting to high gain, nonlinear control theory, or other complicated mathematical tools.

\section{Acknowledgements}

This work was supported in part by Science Foundation of Aeronautics (20080769006, 20100169002), Graduate Innovation Program of Jiangsu Province (CX10B_077Z), and Sci- 
entific Research Foundation of Graduate School of Southeast University. J. Yang would like to thank the China Scholarship Council (CSC) for supporting his visiting in the UK. He also acknowledges the Department of Aeronautical and Automotive Engineering for hosting his research at Loughborough University.

\section{References}

[1] Lee, S.-Y., Lee, J.-I. and Ha, I.-J. Nonlinear autopilot for high maneuverability of bank-to-turn missiles. IEEE Trans. Aerosp. Electron. Syst., 2001, 37(4), 1236-1253.

[2] Uang, H.-J. and Chen, B.-S. Robust adaptive optimal tracking design for uncertain missile systems: a fuzzy approach. Fuzzy Sets Syst., 2002, 126(1), 63-87.

[3] Lin, C.-F., Cloutier, J. R. and Evers, J. H. High-performance, robust, bank-to-turn missile autopilot design. J. Guid. Control Dyn., 1995, 18(1), 46-53.

[4] Lin, C.-K. and Wang S.-D. An adaptive $H_{\infty}$ controller design for bank-to-turn missiles using ridge gaussian neural networks. IEEE Transactions on Neural Networks, 2004, 15(6): 1507-1516.

[5] Lin C.-K. Mixed $H_{2} / H_{\infty}$ autopilot design of bank-to-turn missiles using fuzzy basis function networks. Fuzzy Sets Syst., 2007, 158(20), 2268-2287.

[6] Whalley, R. and Ebrahimi, M. The dynamic analysis of mechanical systems and structures with large parameter variations. Proc. Inst. Mech. Eng. Part G-J. Aerosp. Eng., 1997, 211(G3), 169-181.

[7] Carter, L. H. and Shamma, J. S. Gain-scheduled bank-to-turn autopilot design using linear parameter varying transformations. J. Guid. Control Dyn., 1996, 19(5), 10561063.

[8] Lee, C. H., Shin, M. H., and Chung, M. J. A design of gain-scheduled control for a linear parameter varying system: an application to flight control . Control Eng. Practice, 2001, 9(1), 11-21.

[9] Kim, M. J., Kwon, W. H. and Kim, Y. H. Autopilot design for bank-to-turn missiles using receding horizon predictive control scheme. J. Guid. Control Dyn., 1997, 20(6), 1248-1254.

[10] Duan, G. R., Wang, H. Q. and Zhang, H. S. Parameter design of smooth switching controller and application for bank-to-turn missiles. Aerospace Control, 2005, 23(2), 41-46. (in Chinese)

[11] Chen, X. S., Yang, J., Li, S. H. and Li, Q. Disturbance observer based multi-variable control of ball mill grinding circuits. J. Process Control, 2009, 19(7), 1205-1213.

[12] Shim, H. and Jo, N. H. An almost necessary and sufficient condition for robust stability of closed-loop system with disturbance observers. Automatica, 2009, 45(1), 296299.

[13] Chen, W.-H. Nonlinear disturbance observer-enhanced dynamic inversion control of 
missiles. J. Guid. Control Dyn., 2003, 26(1), 161-166.

[14] Guo, L. and Chen, W.-H. Disturbance attenuation and rejection for systems with nonlinearity via DOBC approach. Int. J. Robust Nonlinear Control, 2005, 15(3), 109125 .

[15] Chen, W.-H., Ballance, D. J., Gawthrop, P. J. and O'Reilly, J. A nonlinear disturbance observer for robotic manipulators. IEEE Trans. Ind. Electron., 2000, 47(4), 932-938.

[16] Komada, S., Machii, N. and Hori, T. Control of redundant manipulators considering order of disturbance observer. IEEE Trans. Ind. Electron., 2000, 47(2), 413-420.

[17] Guo, L. and Tomizuka, M. High-speed and high-precision motion control with an optimal hybrid feedforward controller. IEEE-ASME Trans. Mechatron., 1997, 2(2), 110-122.

[18] Liu, T.-H., Lee, Y.-C. and Chung, Y.-H. Adaptive controller design for a linear motor control system. IEEE Trans. Aerosp. Electron. Syst., 2004, 40(2), 601-616.

[19] Yang, J., Li, S. H., Chen, X. S. and Li, Q. Disturbance rejection of ball mill grinding circuits using DOB and MPC. Powder Technol., 2010, 198(2), 219-228.

[20] Wu, S. N., Sun, X. Y., Sun, Z. W. and Wu, X. D. Sliding-mode control for staringmode spacecraft using a disturbance observer. Proc. Inst. Mech. Eng. Part G-J. Aerosp. Eng., 2010, 224(G2), 215-224.

[21] Wu, X. J. and Guo, L. Composite disturbance-observer-based control and H-infinity control for complex continuous models. Int. J. Robust Nonlinear Control, 2010, 20(1), 106-118.

[22] Barmish, B. R. and Leitmann, G. On ultimate boundedness control of uncertain systems in the absence of matching assumptions. IEEE Trans. Autom. Control, 1982, 27(1), 153-158.

[23] Tan, F. and Duan G. R. Global stabilizing controller design for linear time-varying systems and its application on BTT missiles. Journal of System Engineering and Electronics, 2008, 19(6), 1178-1184.

[24] Fu, L.-C., Chang, W.-D., Yang, J.-H. and Kuo, T.-S. Adaptive robust bank-to-turn missile autopilot design using neural networks. J. Guid. Control Dyn., 1997, 20(2), 346-354.

[25] Yang, J., Zolotas, A., Chen, W.-H., Michail, K. and Li, S. H. Robust control of a nonlinear MAGLEV suspension system with mismatched uncertainties via DOBC approach. Submitted to ISA Transactions.

[26] Mehrabian, A. R. and Roshanian, J. Skid-to-turn missile autopilot design using scheduled eigenstructure assignment technique. Proc. Inst. Mech. Eng. Part G-J. Aerosp. Eng., 2006, 220(G3), 225-239. 


\section{APPENDIX}

\section{Notation}

$a_{i}, b_{i}(i=1, \cdots, 5), e_{1}, e_{2} \quad$ aerodynamic parameters of the missile systems
$\mathbf{A}, \mathbf{B}_{u}, \mathbf{B}_{d}, \mathbf{C}, \mathbf{D}_{u}, \mathbf{D}_{d}$
system matrixes of the missile plant
$\mathbf{A}_{n}, \mathbf{B}_{n}, \mathbf{B}_{l d}, \mathbf{C}_{n}, \mathbf{D}_{n}, \mathbf{D}_{l d}$
system matrixes of the nominal missile model
$\boldsymbol{d}_{x}, \boldsymbol{d}_{y}$
external disturbance vectors on states and outputs
$\boldsymbol{d}_{l x}, \boldsymbol{d}_{l y}$
$\hat{\boldsymbol{d}}_{l x}, \hat{\boldsymbol{d}}_{l y}$
$d_{\omega_{z}}, d_{\alpha}, d_{\omega_{y}}, d_{\beta}$
lumped disturbances on states and outputs
estimates of the lumped disturbances on states and outputs
$d_{n_{z}}, d_{n_{y}}$
$\boldsymbol{e}_{d_{l x}}, \boldsymbol{e}_{d_{l y}}$
$g$
I
external disturbances on states
external disturbances on outputs
estimation errors of the lumped disturbances on states and outputs
gravity acceleration
identity matrix with appropriate dimensions
$J_{x}, J_{y}, J_{z}$
roll,yaw and pitch moments of inertia
$\mathbf{K}_{d_{x}}, \mathbf{K}_{d_{y}}$
$\mathbf{K}_{x}$
disturbance compensation gains for the state and output parts
$\mathbf{L}$
$n_{y}, n_{z}$
$\mathbf{O}$
feedback control gain
gain matrix of the disturbance observer
overloads on the side and normal directions
zero matrix with appropriate dimensions
$v_{t}$
instantaneous speed of the missile
$z$
auxiliary vector of the disturbance observer
$\alpha$
angle-of-attack
$\beta$
sideslip angle
$\delta_{y}, \delta_{z}$
yaw and pitch control deflection angles
$\omega_{x}, \omega_{y}, \omega_{z}$
roll, yaw, and pitch angular rates 


\section{List of Figures}

Number Caption Page

1 Block diagram of the proposed DOBRC method for the BTT missile system 11

2 Output responses in the presence of external disturbances 12

3 Input time histories in the presence of external disturbances 12

$4 \quad$ State responses in the presence of external disturbances 13

$\begin{array}{lll}5 & \text { Output responses in the case of model uncertainties } & 14\end{array}$

6 Time histories of actuators in the case of model uncertainties 15

$7 \quad$ State responses in the case of model uncertainties 16 